\title{
Comparison of Experimental and Numerically Simulated Fatigue Crack Propagation *
}

\author{
Maigefeireti MAITIREYIMU**, Masanori KIKUCHI** and Mamtimin GENI*** \\ ${ }^{* *}$ Department of Mechanical Engineering, \\ Faculty of Science and Technology, \\ Tokyo University of Science \\ 2641 Yamazaki, Noda, Chiba 278-8510, Japan \\ E-mail: mahpiret@me.noda.tus.ac.jp \\ *** School of Mechanical Engineering, Xinjiang University \\ 21 Youhao Beilu, Urumqi, 830008, China
}

\begin{abstract}
The objective of this study is to present a methodology that can properly simulate three-dimensional fatigue crack propagation with consideration of the crack closure effect. To simulate fatigue crack growth in three-dimensional elastic structures, the superposition finite element method (S-FEM) is employed. A local model is used to represent the near crack area to overcome difficulties that appear in the process of re-meshing the FE model during crack propagation; therefore, many complex crack geometries that may be generated under mixed mode conditions can be easily modeled. Considering the plasticity-induced crack closure effect, elastic-plastic analysis was first conducted to study the behavior of the crack closure effect along the crack tip. Normalized crack opening level along the direction of thickness was obtained and applied to simulations. Experiments on single edge notch specimens under Mode I, Mode I+II or Mode I+II +III loading conditions were conducted to verify the method. Experimental results related to crack growth direction, crack shape and fatigue life were compared with simulation results. Better predictions on crack path were shown. Significant improvement of crack shape evolution in the simulation model was obtained considering the crack closure effect.
\end{abstract}

Key words: Fatigue Crack, Crack Closure Effect, Mixed-Mode, S-FEM

\section{Introduction}

Fatigue cracks are considered to be a main cause of disastrous accidents. Failure analysis is mainly based on experimental analysis, and recently, failure simulation has become a novel and supplementary approach to failure analysis. Failure simulations can represent how accidents occur under realistic conditions and with the development in fracture mechanics, the accuracy of simulation is highly emphasized.

Fatigue crack growth simulation consists of two key parts: numerical analysis and mesh generation. A crack in a real structure is always under mixed-mode condition and grows into a complex geometry. Modeling arbitrary crack geometry and maintaining good accuracy at the crack tip has a more practical meaning. Many numerical methods have been applied in this field, such as the finite element method (FEM), the boundary element method (BEM), the extended finite element method (X-FEM) and free mesh method. At present, FEM can be used to easily simulate two-dimensional problems of plural interactive cracks $^{(1)}$. Three-dimensional problems can also be solved using the FEM, by taking advantage of the sub-modeling technique to maintain accuracy around the crack tip. This method have been 
applied to some failure analyses ${ }^{(2)(3)}$. BEM and X-FEM are used, because of their less complex modeling ${ }^{(4)(5)}$. There is another method that is in the frame of FE, superposition FEM (S-FEM), in which the local field near the crack is modeled independently from the global structure. In this case, the local model can be arbitrarily generated according to the crack geometry, as long as it is not out of the entire structure field. Besides, 20-node hexahedral element can be used to keep high accuracy, which is difficult to handle in re-meshing procedure using general FEM. S-FEM performs well not only for two-dimensional problems, but also three-dimensional problems. Multiple crack problems have been presented by modeling each crack as a local mesh ${ }^{(6)}$, and this method is used in this study, due to its ease of use.

Many experimental observations have indicated that crack retardation or acceleration may occur in some cases. Elber ${ }^{(7)}$ found that a crack closed before minimum load was approached, due to plastic deformation around the crack tip, which is known as plasticity induced crack closure and is assumed to be for a cause of crack growth retardation. Numerical simulations have provided a comparatively clear understanding of the crack closure effect ${ }^{(8)(9)}$. In the $1970 \mathrm{~s}$, Chermahini et al. ${ }^{(10)}$ conducted a three-dimensional elastic-plastic analysis on a middle-crack model with different thickness. After that, many other three-dimensional elastic-plastic analyses on plasticity induced crack closure have indicated that the crack closure level varied along the thickness direction, and this may lead to a curved crack tip shape ${ }^{(11)(12)}$.

The objective of this study is to present a S-FEM-based fatigue crack growth simulation method that also considers the plasticity induced crack closure effect. This method was then verified by comparison with experimental results. Experiments were conducted using specimens with through-thickness notches under Mode I, Mode I+II or Mode I+II+III loading conditions. Materials and testing procedure are described first, followed by theory and procedural methods. Elastic-plastic analyses were conducted and a normalized crack opening level along the thickness direction was obtained and incorporated into the simulation method. Simulations considering crack closure were carried out on problems that corresponded to experiments. In $\S 5$, both experimental and simulation results are presented and compared to demonstrate the significance of this simulation method.

\section{Material and Testing Procedure}

General-purpose A2017-T351 aluminum alloy was used as the test specimen in this study. This all is commonly used in rivets, general structural applications and transportations, due to its high strength. Tables 1 and 2 present the chemical composition and mechanical properties of this alloy, respectively. Each specimen had a notch at one side with $0.2 \mathrm{~mm}$ diameter notch tip machined by electric wire cut machine. The fatigue life prediction is based on linear elastic fracture mechanics. And Paris law expressed in Eq. (1) is employed. Figure 1 indicates the relation between crack growth rate and the stress intensity factor (SIF) range at stress ratio $\mathrm{R}=0.1$. $\mathrm{C}$ and $\mathrm{n}$ are material constants for A2017-T351, and are $2.0 \times 10^{-11}$ and 3.43 , respectively.

$$
\frac{d a}{d N}=C(\Delta K)^{n}
$$

Table 1. Chemical composition of A2017-T351.

\begin{tabular}{cllllllll}
\hline Element & $\mathrm{Si}$ & $\mathrm{Fe}$ & $\mathrm{Cu}$ & $\mathrm{Mn}$ & $\mathrm{Mg}$ & $\mathrm{Cr}$ & $\mathrm{Zn}$ & $\mathrm{Ti}$ \\
\hline Content $(\%)$ & 0.71 & 0.29 & 3.64 & 0.67 & 0.65 & 0.03 & 0.11 & 0.03 \\
\hline
\end{tabular}


Table 2. Mechanical properties of A2017-T351.

\begin{tabular}{cccc}
\hline $\begin{array}{c}\text { Tensile Strength } \\
(\mathrm{MPa})\end{array}$ & $\begin{array}{c}\text { Yield strength } \\
(\mathrm{MPa})\end{array}$ & $\begin{array}{c}\text { Elongation } \\
(\%)\end{array}$ & $\begin{array}{c}\text { Young's Modulus } \\
(\mathrm{MPa})\end{array}$ \\
\hline 435 & 333 & 20 & 70600 \\
\hline
\end{tabular}

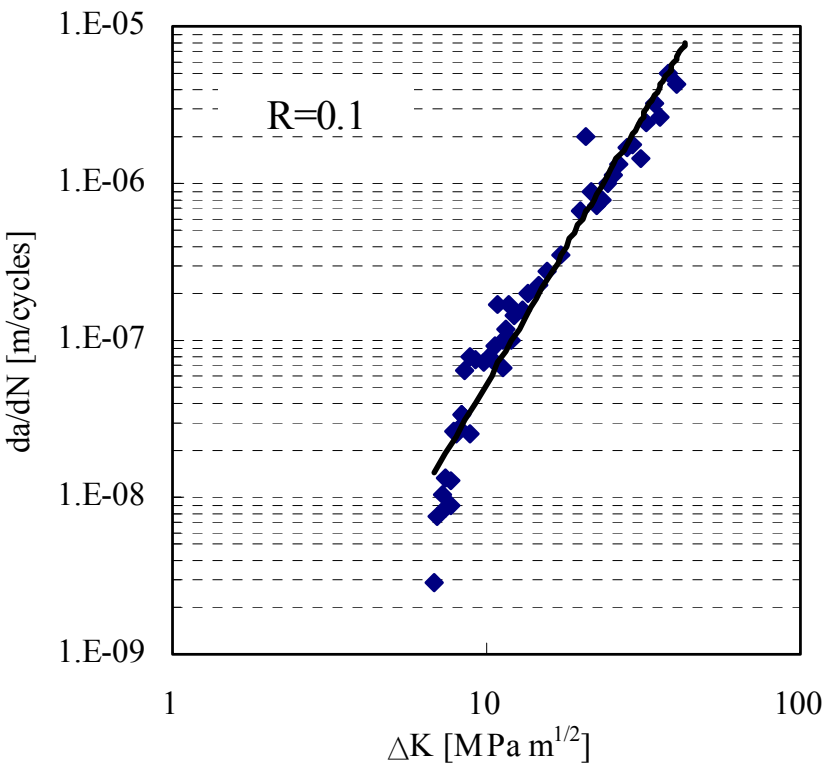

Fig. 1. Relation between crack growth rate and the stress intensity factor range for A2017-T351.

Experiments were performed using a servo-hydraulic machine. The specimens were loaded under three-point bending. Tests were carried out at room temperature with a frequency of $20 \mathrm{~Hz}, \mathrm{R}=0.1$. Three different cases were considered, the geometry and loading conditions of which are illustrated in Figs. 2 4. Three tests were performed for each case with same specimen under identical loading condition. Case 1 was a pure mode I test under cyclic load with $8 \mathrm{kN}$ of maximum load. Case 2 was designed as a Mode I+II combined problem with a Mode II to Mode I ratio of 0.12. In this case, a pre-crack from the notch was introduced at first under Mode I loading condition. During this procedure, it is hard to control and make cracks to extend same length from notches in each test. The average pre-crack length, $2 \mathrm{~mm}$, is labeled in Fig. 3 and detail information is provided in Table 3. The specimen was then moved $20 \mathrm{~mm}$ rightwards from the upper loading pin and subjected to a cyclic load with $6 \mathrm{kN}$ of maximum load. In these two tests, $10 \mathrm{~mm}$ thick specimens were used. The third test was performed on a $20 \mathrm{~mm}$ thick specimen with a notch positioned at $45^{\circ}$ to the specimen surface. No pre-crack was introduced in this case, due to difficulties in forming the pre-crack. In this case, the maximum load was $12 \mathrm{kN}$.

In each case, the beach mark method was employed to check the crack growth rate and to record the crack shape by adjustment of the stress ratio to 0.8 while the maximum load was kept constant. For clarity, $10^{5}$ load cycles were required for one beach mark, and beach marks were measured from fracture surfaces images acquired for graphical analysis. Crack length was observed using a CCD camera with $200 \times$ zoom lens. The direction of crack growth was measured using a CCD laser measurement system.

Table 3. Defect length after pre-crack in each test for case 2.

\begin{tabular}{cccc}
\hline Length $(\mathrm{mm})$ & Test-1 & Test-2 & Test-3 \\
\hline At the middle & 21.74 & 20.71 & 20.77 \\
At the surface & 22.57 & 21.31 & 21.35 \\
\hline
\end{tabular}




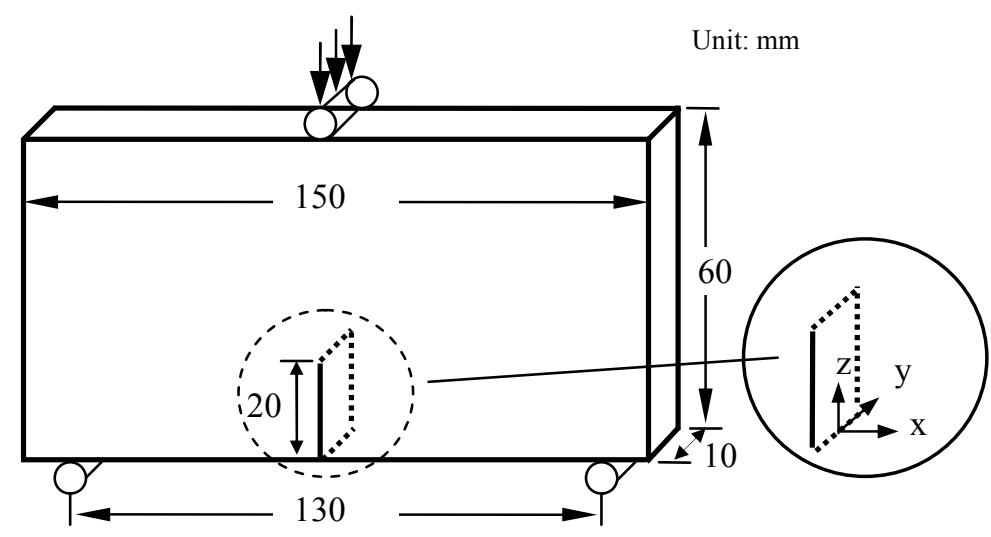

Fig. 2. Schematic illustration of Case 1 test.

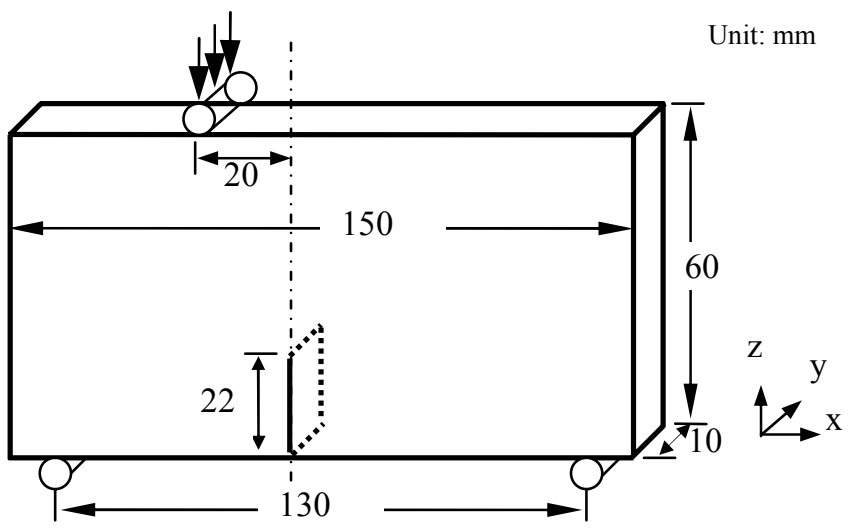

Fig. 3. Schematic illustration of Case 2 test.

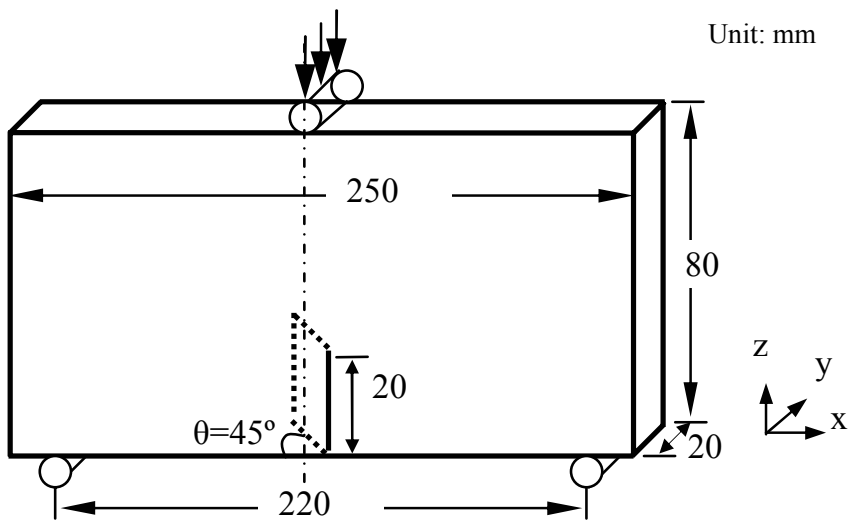

Fig. 4. Schematic illustration of Case 3 test.

The reference coordinate system used in the following sections is illustrated in Fig. 2. The coordinate origin is positioned at the middle point of the bottom plane of the specimen. Coordinate axis $y$ is in the thickness direction. In the mixed mode case, the crack path was viewed along the $y$ direction. The crack shape was plotted by projecting the fracture surface on the $y-z$ plane.

\section{Numerical Analysis}

\subsection{S-FEM}

S-FEM was originally proposed by Fish and Markolefas ${ }^{(13)}$ as the global-local method used to resolve the high gradient laminate plate problem. The flexibility of this method involves the use of computer resources to analyze the key part of a complex problem, such 
as the vicinity of holes, inclusions and cracks. It extracts the key areas from the entire structure and combines them with a mathematical relationship.

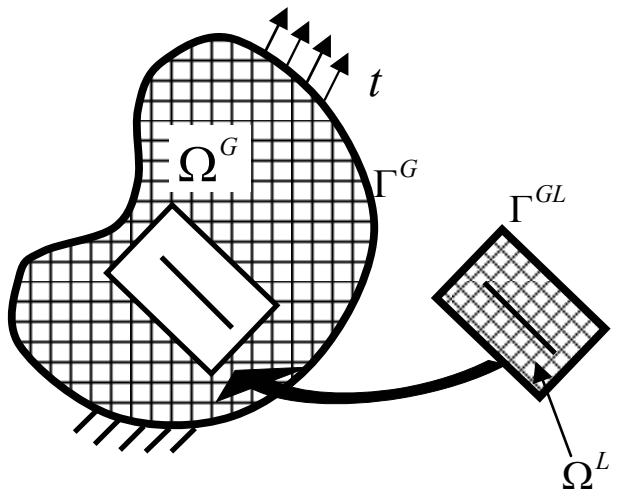

Fig. 5. Crack problem illustrated by the S-FEM model

This method defines the total problem using two models; the global model and local model, which are expressed as $\Omega^{G}$ and $\Omega^{L}$, respectively. These two models should be continuous at the boundary $\Gamma^{G L}$. In this case, the displacements are expressed as:

$$
u_{i}= \begin{cases}u_{i}^{G} & i \in \Omega_{G}-\Omega_{L} \\ u_{i}^{G}+u_{i}^{L} & i \in \Omega_{L} \\ u_{i}^{L}=0 & i \in \Gamma^{G L}\end{cases}
$$

The variation of displacements can be written in the same way. The displacement and variation of displacement are then inserted into the virtual work principle. The S-FEM equation is derived as Eq. (3), and the matrix form is given in Eq. (4)

In Eq. (4), the stiffness matrix is symmetric and consists of four parts, in which $\left[K^{G L}\right]=\left[K^{L G}\right]$. These two parts are expressed by the relationship between the two models

$$
\begin{aligned}
& \int_{\Omega^{G}} \delta u_{i, j}^{G} D_{i j k l} u_{k, l}^{G} d \Omega+\int_{\Omega^{L}} \delta u_{i, j}^{G} D_{i j k l} u_{k, l}^{L} d \Omega \\
& +\int_{\Omega^{L}} \delta u_{i, j}^{L} D_{i j k l^{u}} u_{k, l}^{G} d \Omega+\int_{\Omega} \delta u_{i, j}^{L} D_{i j k l} u_{k, l}^{L} d \Omega=\int_{\Gamma^{t}} \delta u_{i}^{G} t_{i} d \Gamma^{t G}
\end{aligned}
$$

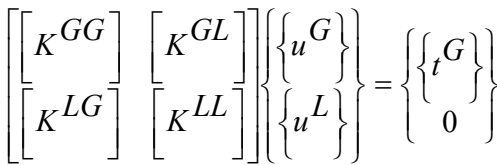

The theoretical details have been previously reported ${ }^{(6)}$.

\subsection{Simulation procedure}

The simulation is based on linear fracture mechanics. Crack growth behavior is discretized into a number of incremental steps. The procedure for the simulation method is illustrated in Fig. 6.

The global model represents the overall structure and neglects cracks, whereas the local model contains cracks. Both models are meshed using 20-node hexahedral elements. Based on these two models, the SIF for Mode I, Mode II and Mode III, which is $\mathrm{K}_{\mathrm{I}}, \mathrm{K}_{\mathrm{II}}$ and $\mathrm{K}_{\mathrm{III}}$ can be obtained by S-FEM calculation combined with the virtual crack closure method $(\mathrm{VCCM})^{(14)}$. Using the VCCM, energy release rates for each mode loading condition are calculated and are then transformed into stress intensity factors considering the plane strain state. 


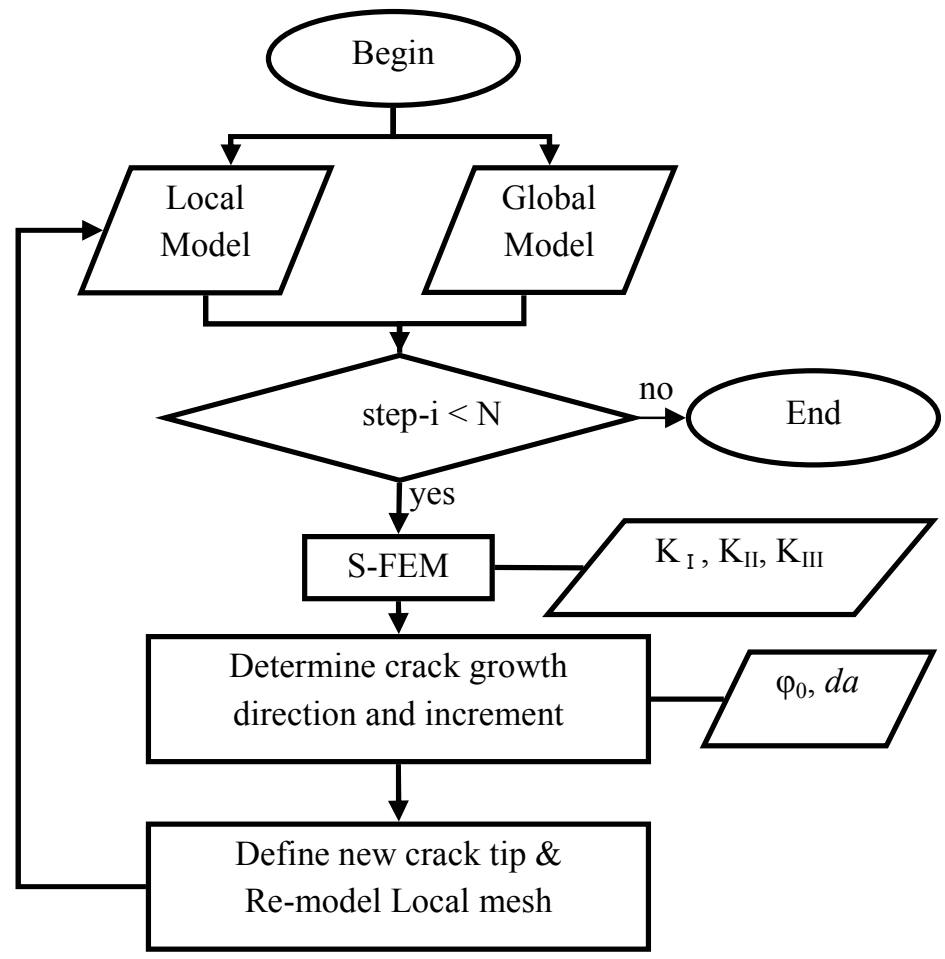

Fig. 6. Procedure for fatigue crack growth simulation by S-FEM.

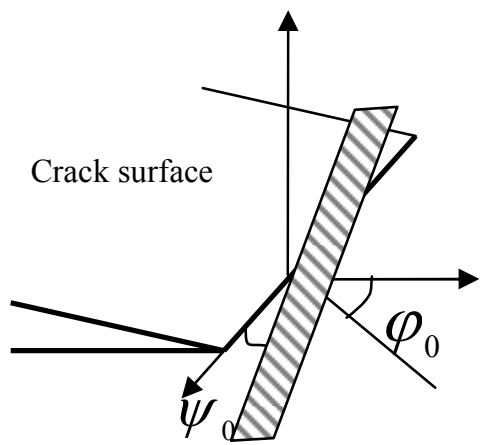

Fig. 7 Local coordination of crack tip

There is always local kinking, $\varphi_{0}$, and local twisting, $\psi_{0}$, of the crack front, which is induced by the Mode II and Mode III portions as illustrated in Fig. 7. Equation (5) contributed by Schollman and Richard ${ }^{(15)}$ is then used to determine the kinking direction of crack growth, where $\varphi_{0}<0^{\circ}$ for $K_{I I}>0$ and $\varphi_{0}>0^{\circ}$ for $K_{I I}<0$. From Fig. 7, it can be considered that small local facets along the crack tip will be created by twisting angle $\psi_{0}$ and irregular crack surface will be formed. Consequently, twisting behavior is difficult to model using FE mesh; therefore, only the kinking angle is taken into account:

$$
\varphi_{0}=\mp\left[140^{\circ} \frac{\left|K_{I I}\right|}{K_{I}+\left|K_{I I}\right|+\left|K_{I I I}\right|}-70^{\circ}\left(\frac{\left|K_{I I}\right|}{K_{I}+\left|K_{I I}\right|+\left|K_{I I I}\right|}\right)^{2}\right] .
$$

The equivalent SIF range for a mixed mode condition can be approximated from each of the Mode components using Eq. (6) ${ }^{(16)}$. For a three-dimensional simulation, the increment at each node, $d a$, on the crack tip should be determined. The maximum increment $d a_{\max }$, at the point corresponding to the maximum SIF range, is set to be a constant value at 
every calculation step. The maximum increment is set according to the size of the element at the crack tip of the initial model. If the model is coarsely meshed, it leads to bad results. And fine mesh will take up more memory. Therefore a balance between keeping good accuracy and saving computer resource is necessary. It is always set to 0.3 or $0.5 \mathrm{~mm}$ in order to keep same element size at each step and maintain stable crack growth in advance. Based on the Paris law, the relationship between the increment of each crack tip node and the maximum crack increment is derived as Eq. (7).

$$
\begin{aligned}
\Delta K_{e q} & =\frac{\Delta K_{I}}{2}+\frac{1}{2} \sqrt{\Delta K_{I}^{2}+4\left(1.115 \Delta K_{I I}\right)^{2}+4\left(\Delta K_{I I I}\right)^{2}} \\
d a_{i} & =d a_{\max } /\left(\Delta K_{e q \max } / \Delta K_{e q i}\right)^{n}
\end{aligned}
$$

A new crack front can then be determined by cubic spline interpolation. According to the new crack front definition, the local model is re-meshed. Elements before the crack tip are moved forwards according to the new crack tip position and new elements are added to fill the gap between the elements. The local model is valid as long as it is restricted to the global model boundaries. This treatment may lead to an increasing amount of elements with crack growth. Therefore, an element combination technique is adopted, at which the area is far from the crack tip. At the next step, a new local mesh is combined with the original global model to carry out the procedure shown in Fig.6 until step number i exceeds the self-defined total step number $\mathrm{N}$.

\subsection{Elastic-Plastic Analyses}

During crack extending, a plastic wake is formed along crack surface. This is caused by large plastic strain, which is developed during loading and is not fully reversed when unloading. This plastic wake near the crack tip results in compressive residual stress and leads to the crack closure effect. Consequently, the driving force for fatigue crack growth is reduced. Plastic induced crack closure commonly occurs in a mode I problem. A symmetric study was employed by taking one fourth of the 3PB model (geometry is shown in Fig. 2). A fine mesh is created near the crack tip shown as Fig. 8. The minimum element size around the crack tip is $0.0206 \mathrm{~mm}$. Remote displacement is applied on the top surface near the middle plane of the model. The size of this area is determined by the Hertz's formula. The stress ratio is 0.1 . The material properties used were same as those shown in Table 2. A bi-linear kinematic hardening model was used for the material model with $\mathrm{H} / \mathrm{E}=0.1$, where $\mathrm{E}$ is Young's modulus and $\mathrm{H}$ is the plastic modulus.

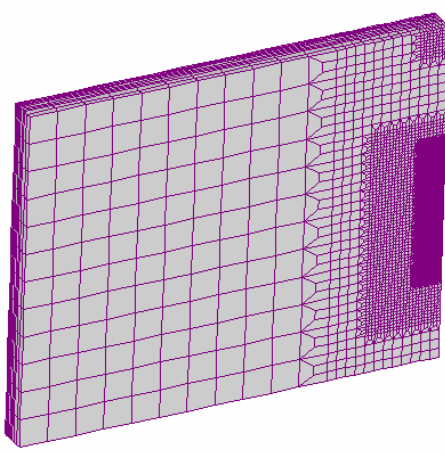

(a)

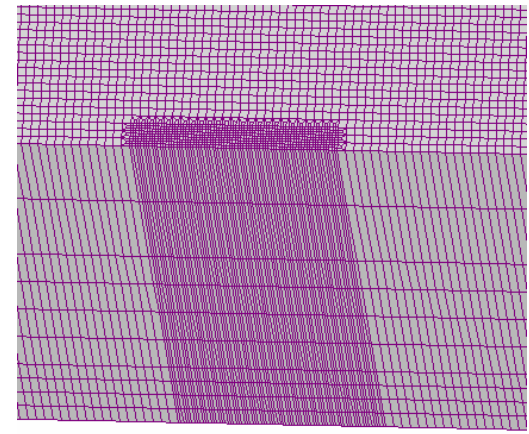

(b)

Fig. 8. Three-point bending FE model; (a) overall, and (b) fine mesh near the crack tip.

The elastic-plastic crack growth simulation was carried out using the node release 
method. The procedure used was as follows. One set of nodes before the crack tip was released from displacement constraints at maximum load $\mathrm{P}_{\max }$. This was followed by unloading; during unloading, the nodal displacements at each node behind the crack tip on the crack surface were monitored. When the opening displacement of one point was equal or below zero, that point was considered as closed and displacement constraints in the opening direction were applied. At the same time, the closing load $\mathrm{P}_{\mathrm{cl}}$ was recorded. After the minimum load $\mathrm{P}_{\min }$, was approached, the load was again increased to maximum load. This procedure was followed for every cycle ${ }^{(17)}$. Usually, crack closure occurs first at the surface, and is then transferred to the inner part. After 10 cycles, crack closure became stationary. The crack opening level was used as a parameter to characterize the crack closure phenomenon. Using Eq. (8), the crack opening level U can be determined, which is the effective portion of the cyclic load to cause crack advance.

$$
U=\frac{P_{\max }-P_{c l}}{P_{\max }-P_{\min }}
$$

The crack opening level distribution along the specimen thickness could be obtained. Different thicknesses, $5 \mathrm{~mm}, 10 \mathrm{~mm}$ and $20 \mathrm{~mm}$, were considered. And a curved crack tip modeled according to beach mark was also taken into consideration. In order to generalize this value for any thickness considered in the simulation, a normalized value, which is the fraction of the crack opening level at one point on the crack front to the middle point value, was plotted against the fraction of the thickness (Fig. 9). Zero on the horizontal ordinate represents the middle plane, while 1 represents the surface plane. The effective cyclic load for crack growth was small at the surface. The effective cyclic load of curved crack front is smaller than straight crack front at the surface. Using a curve fitting technique, Eq. (9) was obtained.

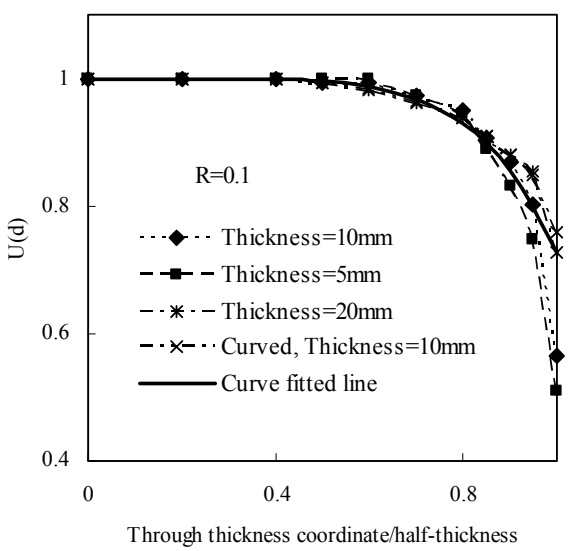

Fig. 9. Normalized opening level change along the normalized thickness in different thickness model and curved crack tip model

$$
U(d)=\frac{U_{i}}{U_{0}}=1-0.27 d^{6}
$$

The parameter $d$ is the fraction of the coordination of one point to the thickness. The ratio of the crack opening level at the surface and at the middle plane is approximately equal to 0.75 . This equation was used to modify the SIF range using Eq. (10).

$$
\Delta K_{e f f i}=\Delta K_{e q i} U(d)
$$


In turn, the increment of each node along the crack front could be obtained using the effective SIF range instead of the equivalent SIF range in Eq. (7).

\section{Results and discussion}

In this section, the experimental and simulation results for the three different tests are presented. In each case, the crack growth direction, crack shape and fatigue life are discussed.

\subsection{Case 1}

Figure 10 shows the fracture surface of the specimen for the Mode I test. Crack tip suffers tensile stress under bending. As a result, crack grows along its original crack plane. The visible lines are beach marks (BM). The straight notch crack front grew into a curved crack front, which is always observed in experiments as the tunneling effect. This indicates that during the early stage of crack growth, the crack tip at the surface grew at a lower speed than that at the inner side. After the curved shape formed, it grew in a self-similar way. The angle that the crack tip intersected with the surface is $106-110^{\circ}$.

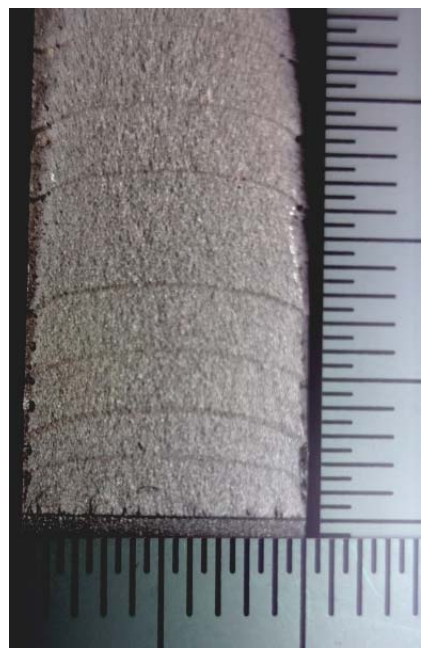

Fig. 10. Fracture surface.

Figure 11 shows the mesh used in the S-FEM. The global and local models were meshed separately. The local model was generated by the means that the area with a certain ligament from the crack tip is selected to ensure accuracy ${ }^{(6)}$. Fine mesh was used around the crack tip, and the crack front was discretized into 20 elements. The local model should be restrained in the field of the global model, as shown as Fig. 12.
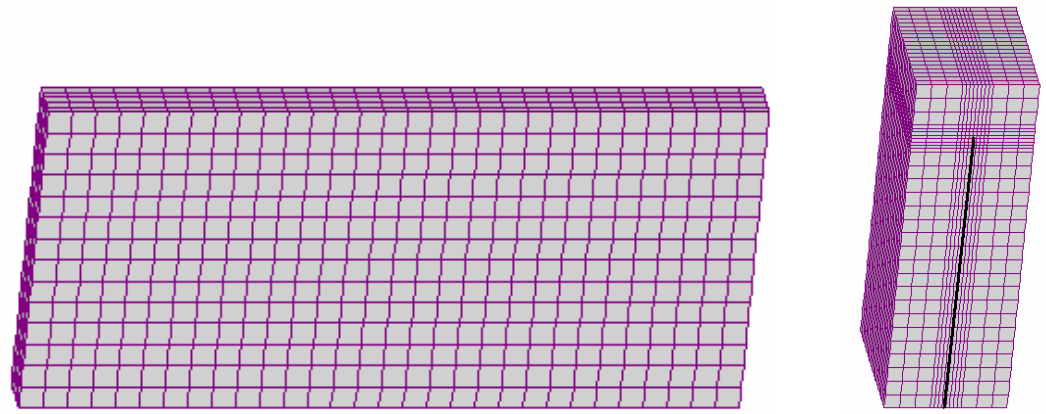

Fig. 11. Simulation model; global model and local model with crack (black line). 


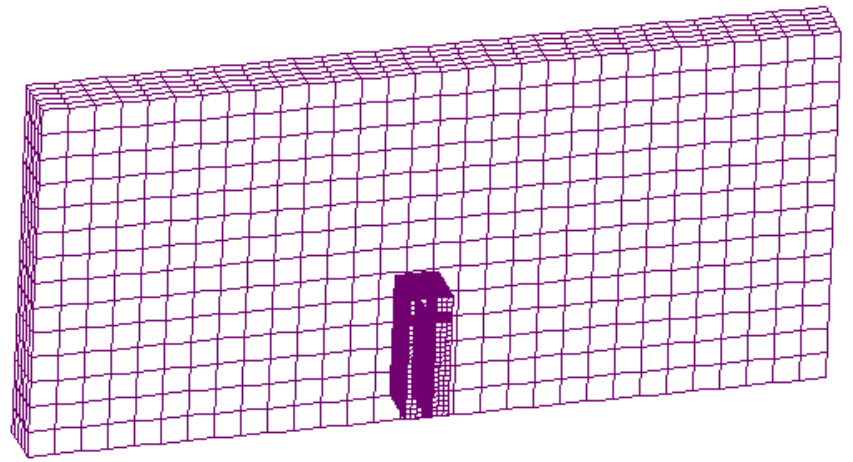

Fig. 12. Local model superimposed on the global model.

The simulation was firstly conducted without consideration of the crack closure effect, resulting in a nearly straight crack shape prediction. There is a large deviation at the near surface part, as shown in Fig. 13(a), compared with the experimental result. The crack closure effect was then considered and the crack opening level was used according to the procedure explained in $\S 4$. The predicted crack shape evolves into the expected curved crack front, and matches the experimental beach marks well, as shown in Fig. 13(b).

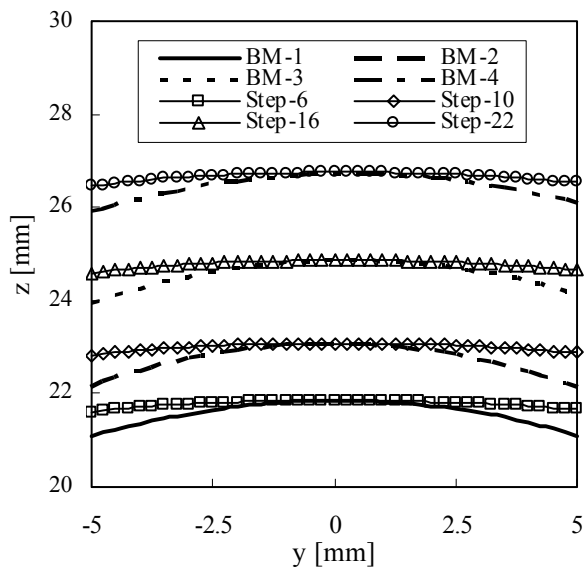

(a)

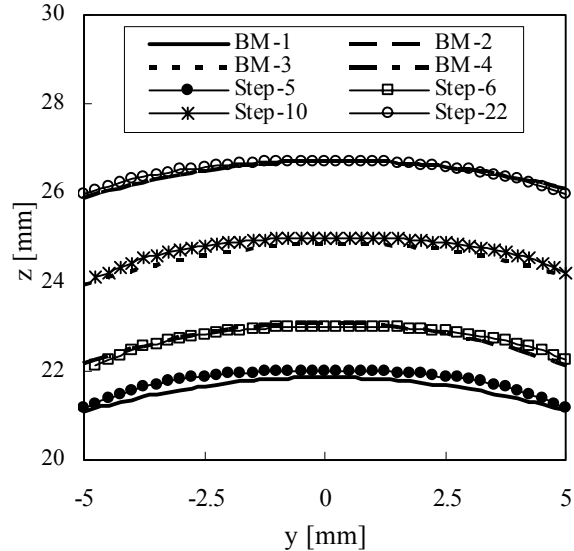

(b)

Fig. 13. Comparison of the simulated and experimental crack shapes: (a) Simulation without consideration of the crack closure effect. (b) Simulation considering the crack closure effect.

Variation of the SIF range along the crack front of a growing crack is shown in Fig. 14(a). Each value of the SIF range at the crack front was evaluated using a set of elements around the crack tip. In these figures, y corresponds to the y coordinate of the element middle node on the crack front. The Mode I SIF at the middle plane is initially large, but when the crack shape evolved into a curved shape, the SIF is large at the surface, which is consistent with that reported in Ref. (18). As a result of the crack closure effect, the stress for crack extension was reduced by surface contact. In this case, the effective SIF range became a constant distribution for the curved crack configuration, due to a varied crack closure level along the crack front, as shown in Fig. 14(b). After the curved crack front converged, the increment at each node on the crack front was almost constant. Consequently, the crack maintained the same crack shape during the remaining fatigue growth, as was found experimentally.

Figure 15 shows the number of load cycles as a function of crack length for a crack growing from the first beach mark to other beach marks. The difference between the predicted and experimental results appeared at the beginning, but finally the total life prediction was in agreement. 


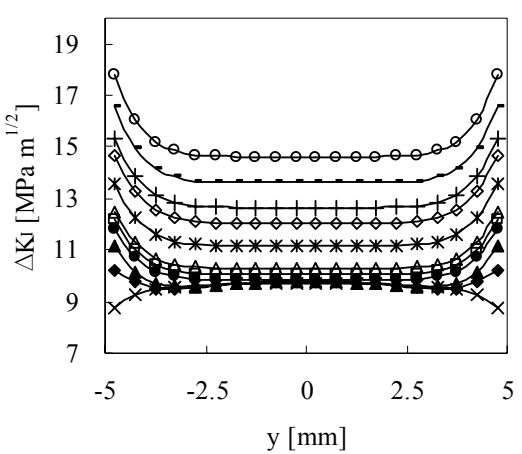

(a)

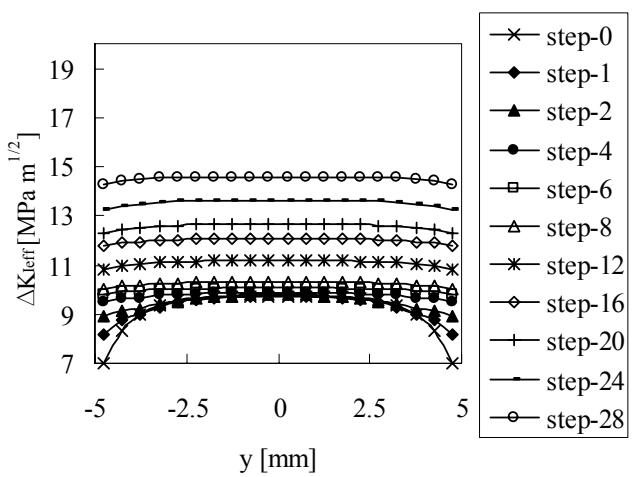

(b)

Fig. 14. Stress intensity factor range during crack growth from the simulation: (a) Stress intensity factor range calculated using the VCCM, and (b) effective stress intensity factor range.

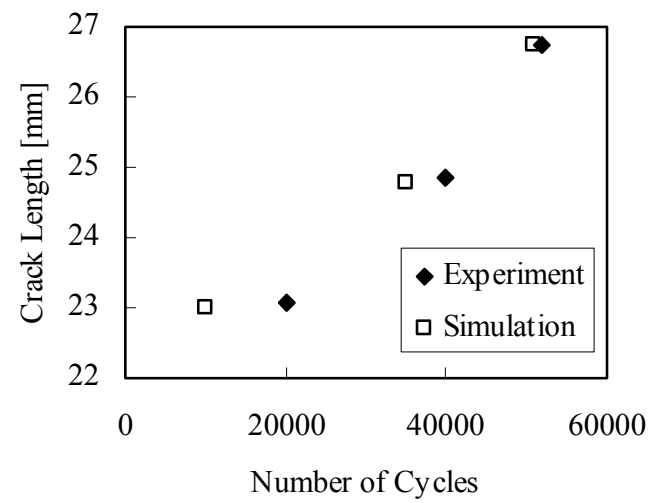

Fig. 15. Comparison of fatigue life.

\subsection{Case 2}

Case 2 is designed as a mode I+II problem. In this case, the crack tip is under a combination of tension and in-plane shearing conditions. The experimental results in Fig. 16 shows that cracks were first inclined at a certain angle and grew along the same direction all the way.
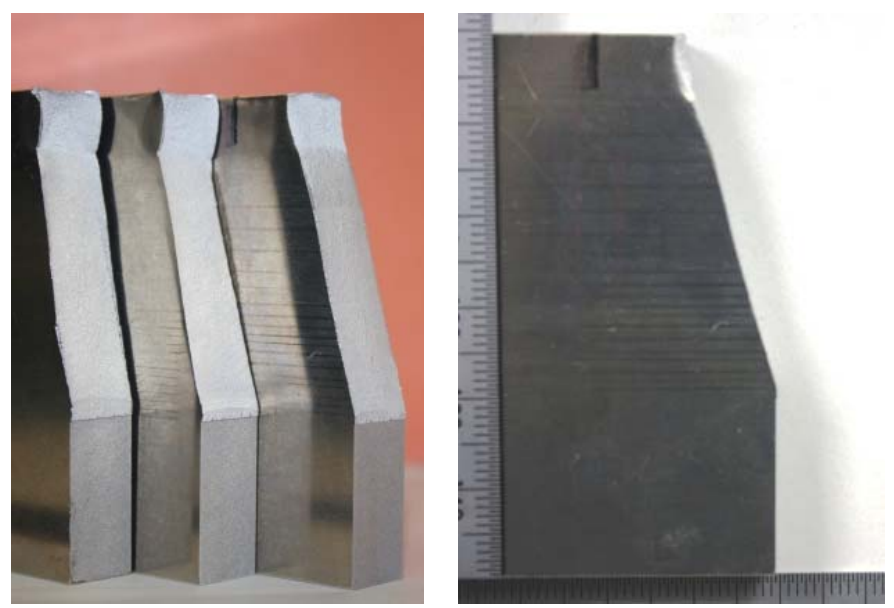

Fig. 16. Photograph of the specimens after failure.

In simulation, the initial local models are generated according to the pre-crack shapes. Figure 17(a) shows SIF distributions for the initial crack configuration in test1. The crack is driven to incline with an angle, due to the Mode II SIF (Fig. 18(b)). Afterwards, the Mode II 
SIF decreased to zero while Mode I increased with crack length (Fig. 17(b)). Mode III SIF is constant to zero. Figure 18(c) shows the crack configuration when the crack length was extended $12 \mathrm{~mm}$ after 40 simulation steps.

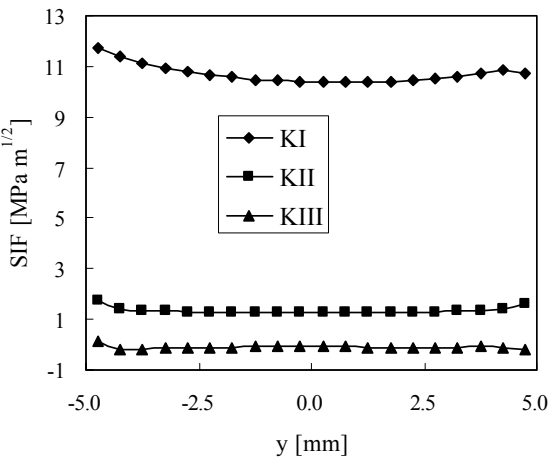

(a)

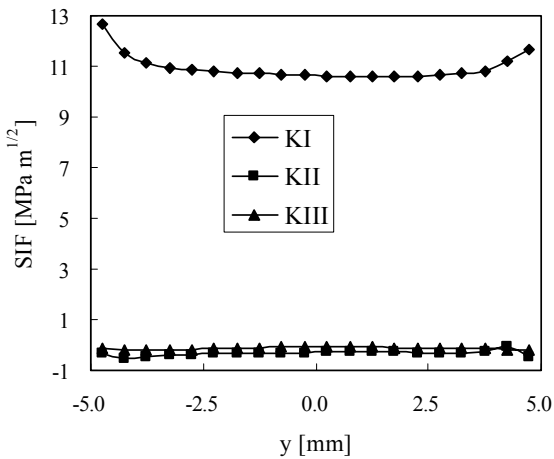

(b)

Fig. 17. Stress intensity factor distributions along the crack tip for the simulation: (a) at initial crack, and (b) at step 1.

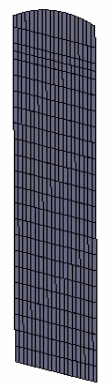

(a)

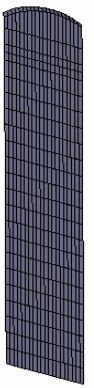

(b)

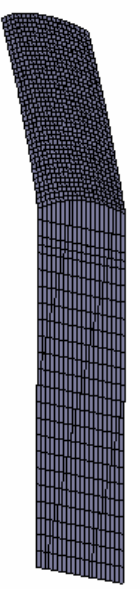

(c)

Fig. 18. Crack surface: (a) at initial crack, (b) at step 1, and (c) at step 40.

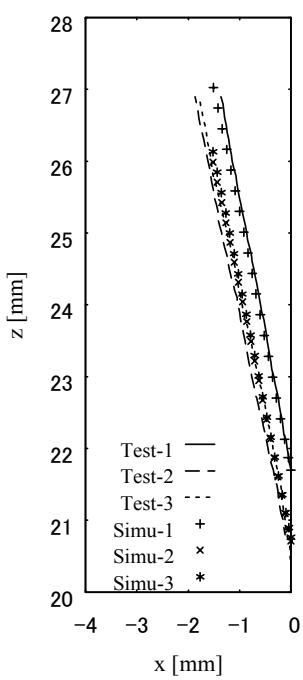

Fig. 19. Crack path at the surface.

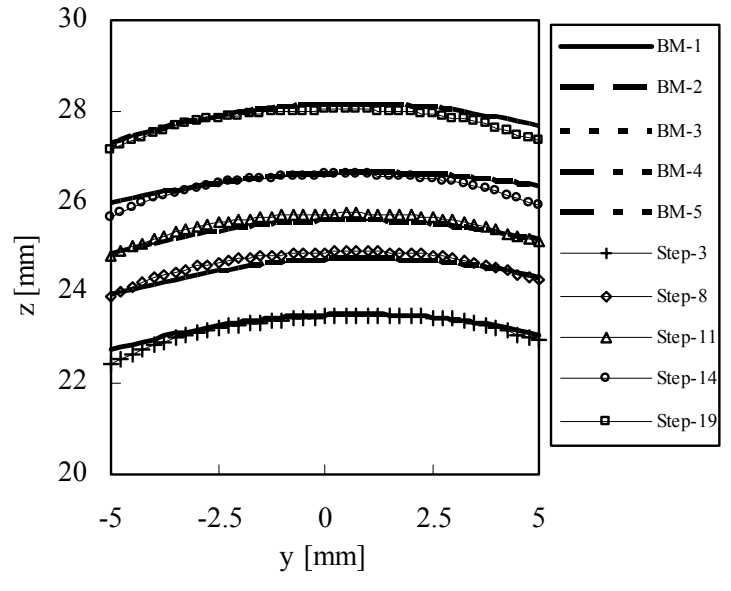

Fig. 20. Comparison of simulated and experimental crack shapes in Test 1. 
Figure 19 shows the predicted crack paths of the crack tips at the surfaces compared with experimental results. The inclined angles predicted in the simulations were in good agreement with the experimental results. The inclined angle predicted in the simulation, using Eq. (5) equals to $14.6^{\circ}$, which is $13.9^{\circ}$ as predicted by the maximum principle stress criterion. After inclination, the crack grew under the Mode I local loading condition. Consequently, the crack shape evolved in the same way as that for Case 1 . The crack shape prediction incorporated with the crack opening level is shown in Fig. 20 by comparison of the beach marks. The fatigue life prediction from the first to last beach mark is 105000 cycles. It took 100000 cycles to experimentally grow this crack length.

\subsection{Case 3}

In this case, the local loading condition was different at each point along the crack tip, because of the asymmetric position of the crack tip in the specimen. The notch tip suffers from overall tensile, in-plane shearing and out-of-plane shearing loading. From Fig. 21, the crack growth behavior in this case can be divided into two stages. At the initial stage, the crack tip changed its position by extending and changing the intersection angle with the specimen surface. The factory roof phenomenon caused by Mode III is observed. Small facets at the root of the notch were created and large distortion of crack surface was formed. Gradually this phenomenon disappeared with crack turning to middle position of specimen. After the crack front was almost normal to the edge, the crack grew into a self-similar extending stage.
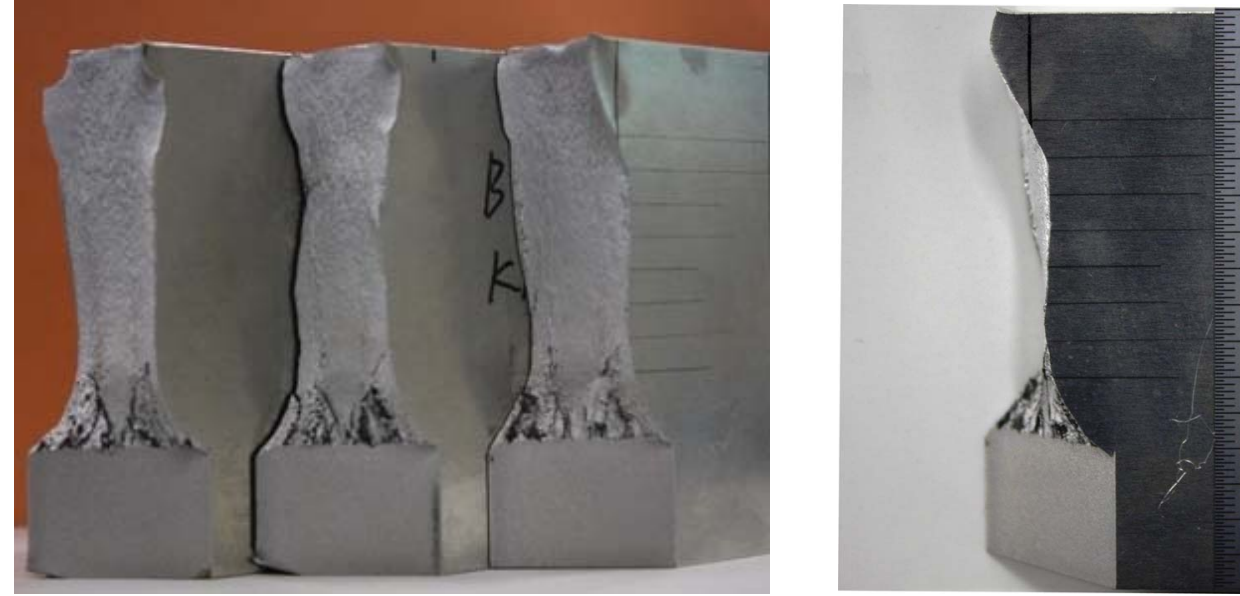

Fig. 21. Photograph of the specimen after failure.

In this case, the advantage of S-FEM for modeling complex crack geometry is demonstrated. Figure 22 shows three results for the SIF distribution along the crack front during crack growth. Even though the mode III effect on crack increment was considered in the simulation, the crack reflection caused by mode III was not taken into consideration. In this case, the factory roof phenomenon could not be simulated. For the initial crack, Mode II and Mode III SIF are significant. Under this condition, the crack front grew toward to the middle plane of the specimen. After 20 simulation steps, the crack configuration transforms, as shown in Fig. 22(b), with Mode II and Mode III stress intensity factors tending towards zero. Finally, the crack also grew into a second stage, as experimentally observed. Mode II and Mode III stress intensity factors became zero, and the crack grew under the Mode I loading condition. 

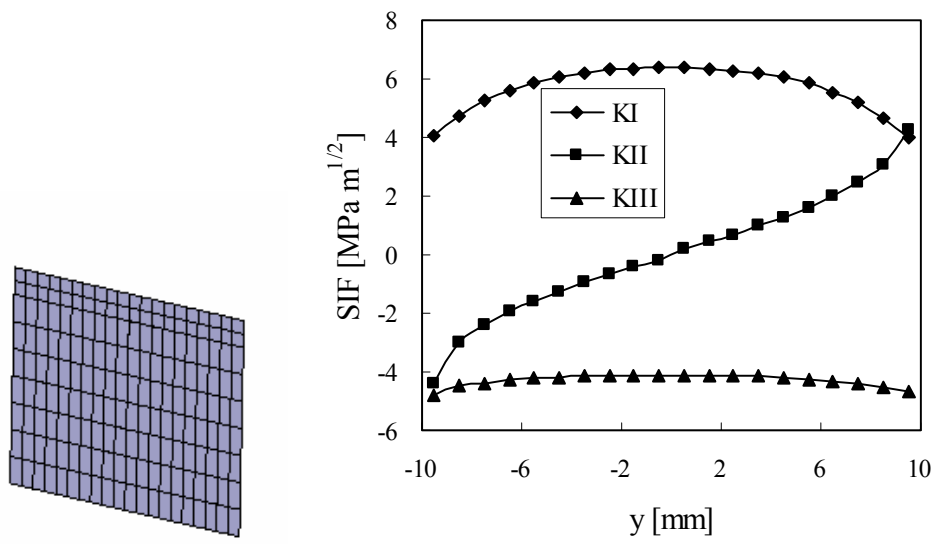

(a)
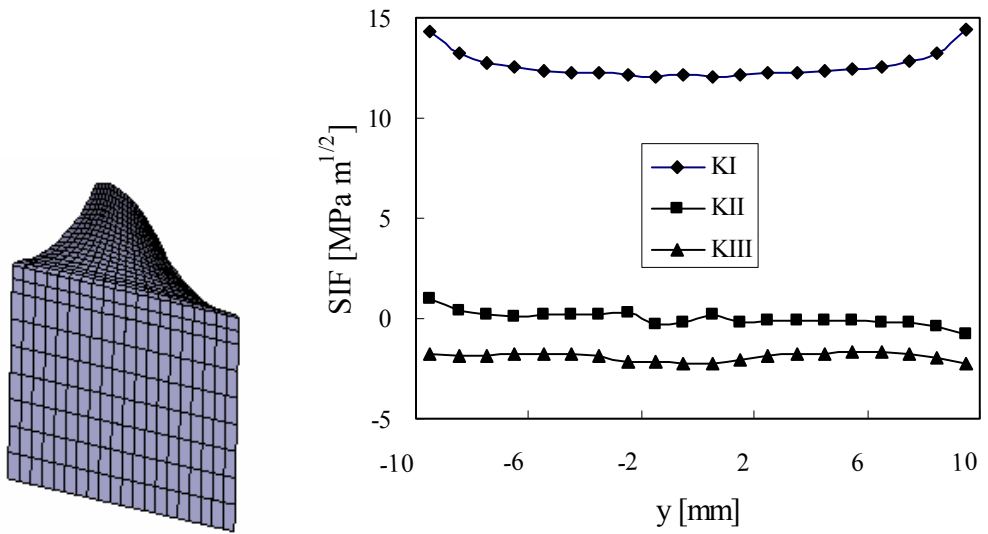

(b)
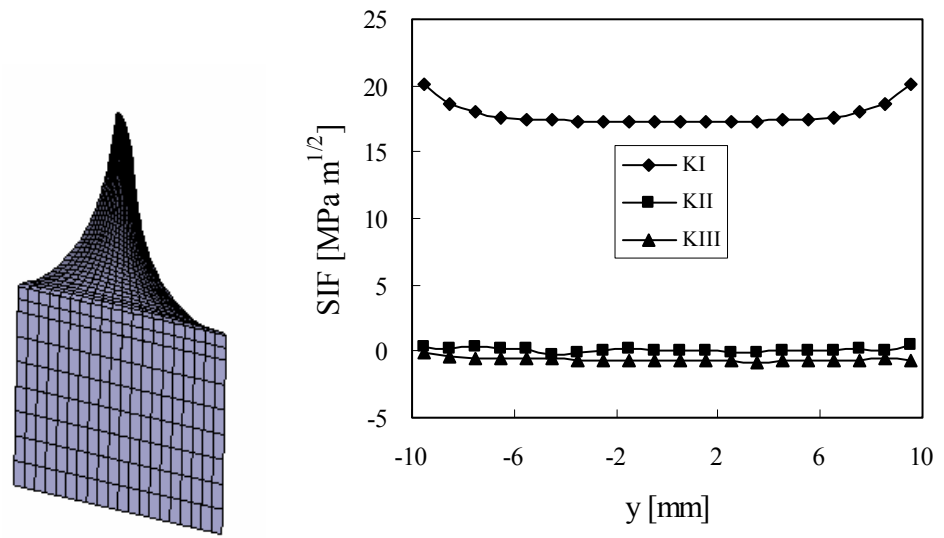

(c)

Fig. 22. Crack surface and stress intensity factor distributions along the crack tip according to the simulation: (a) at initial crack, (b) at step 20, and (c) at step 40.

The predicted crack paths at the surface and middle plane were compared with the experimental results shown in Fig. 23. From the comparison, it is found that the crack at surface in experiments curved larger than in simulation. In the middle side, crack tip extended straightly upwards both in experiments and simulation. The pure mode I condition cannot be precisely achieved at the second stage shown in the experimental results. Although there is small deviation, the trend of crack growth was well predicted. Therefore, the direction prediction of crack growth without considering the Mode III effect is 
acceptable. The twisting behavior can be represented after the Mode III is well understood and will be studied in the future. In particular, the crack shape evolution is still well predicted for different thickness using a normalized crack opening level, as shown in Fig. 24.

Crack growth propagation at the initial stage required a large amount of loading cycles, because the unsmooth surface retarded crack growth rate. Besides the factory roof phenomenon, 40000 cycles were required for the crack to grow from the first beach mark to the last. The simulation provided a predicted value of 34000 cycles.

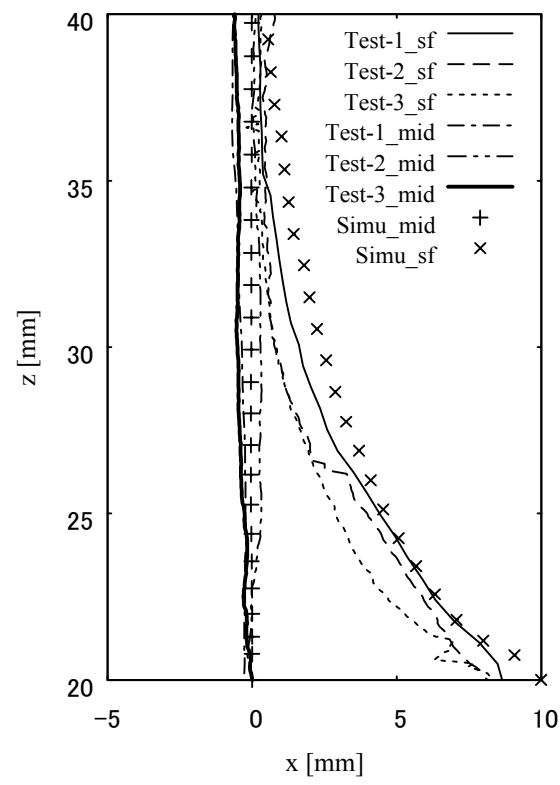

Fig. 23. Crack path at the surface and middle plane.

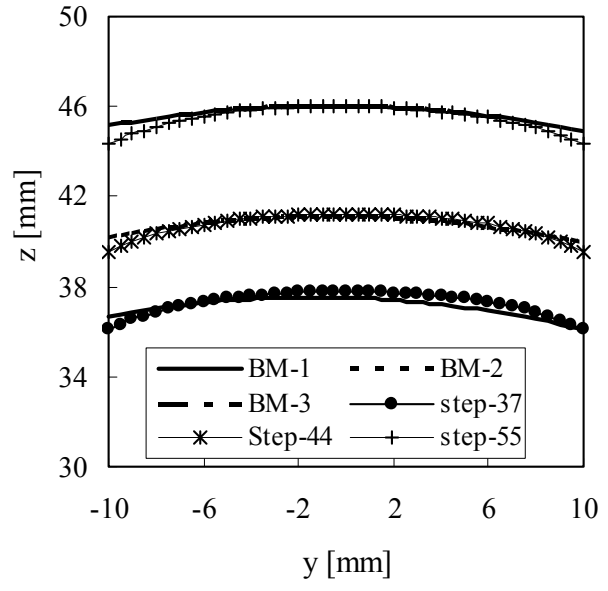

Fig. 24. Comparison of the simulated and experimental crack shapes.

\section{Conclusion}

A three-dimensional fatigue crack propagation simulation method using S-FEM was presented. The crack closure effect was considered in the simulation. Through-thickness crack problems under different mode conditions were investigated both experimentally and by simulation. The crack growth direction, crack shape and crack fatigue life results were compared. Analysis of both the experimental and simulation results led to the following conclusions:

1. Crack growth behavior of complex geometry under mixed mode conditions can be easily simulated using S-FEM.

2. In each case, Mode I fatigue crack growing was always predominant in all cases. Cracks always grow in a self-similar way eventually. Crack growth predictions affected by mode II and mode III conditions were well modeled by the simulation and were consistent with the experimental results.

3. The crack opening level variation model was applied to not only mode I, but also to a mixed mode problem, and both cases appeared to be valid. Crack tunneling is predicted, as expected.

4. Simulation and experimental results were in good agreement. The simulations also provided an well prediction of fatigue crack life.

\section{References}

(1) Yates, J. R., Zanganeh, M., Tomlinson, R. A., Brown, M. W., and Diaz Garrido, F. A., Crack 
path under mixed mode loading, Engineering Fracture Mechanics, Vol. 75 (2008), pp. 319-330.

(2) Richard, H. A., Sander, M., Fulland, M., and Kullmer, G., Development of fatigue crack growth in real structures, Engineering Fracture Mechanics, Vol. 75 (2008), pp. 331-340.

(3) Citarella, R., and Buchholz, F. G., Comparison of crack growth simulation by DBEM and FEM for SEN-specimens undergoing torsion or bending loading, Engineering Fracture Mechanics, Vol. 75, No. 3-4 (2008), pp. 489-509.

(4) Mellings, S., Baynham, J., and Adey, R. A., Automatic crack growth prediction in rails with BEM, Engineering Fracture Mechanics, Vol. 72, No. 2 (2005), pp. 309-318.

(5) Karihaloo, B. L., and Xiao, Q. Z., Modeling of stationary and growing cracks in FE framework without remeshing: a state-of-the-art review, Computers \& Structures, Vol. 81, No. 3 (2003), pp. 119-129.

(6) Okada, H., Endoh, S., and Kikuchi, M., On Fracture Analysis using an Element Overlay Technique, Engineering Fracture Mechanics, Vol. 72 (2005), pp. 773-789.

(7) Elber, W., Fatigue crack closure under cyclic tension, Engineering Fracture Mechanics, Vol. 2 (1970), pp. 37-45.

(8) Antunes, F. V., and Rodrigues, D. M., Numerical simulation of plasticity induced crack closure: Identification and discussion of parameters, Engineering Fracture Mechanics, Vol. 75 (2008), pp. 3101-3120.

(9) Jiang, Y. Y., Feng, M. L., and Ding F., A reexamination of plasticity-induced crack closure in fatigue crack propagation, International Journal of Plasticity, Vol. 21 (2005), pp. 1720-1740.

(10) Chermahini, R. G., Shivakumar, K. N., Newman, J. C., Jr, and Blom, A. F., Three-Dimensional Aspect of Plasticity-Induced Fatigue Crack Closure, Engineering Fracture Mechanics, Vol. 34, No. 2 (1989), pp. 393-401.

(11) Gonzalez-Herrera, A., and Zapatero, J., Tri-dimensional numerical modeling of plasticity induced fatigue crack closure, Engineering Fracture Mechanics, Vol. 75 (2008), pp. 4513-4528.

(12) Solanki, K., Daniewicz, S. R., and Newman, Jr., J. C., Finite element analysis of plasticity-induced fatigue crack closure: an overview, Engineering Fracture Mechanics, Vol. 71 (2004), pp. 149-171.

(13) Fish, J., and Markolefas, S., The S-version of the Finite Element Method for Multilayer Laminates, International Journal for Numerical Methods in Engineering, Vol. 33 (1992), pp. 1081-1105.

(14) Okada, H., Higashi, M., Kikuchi, M., Fukui, Y., and Kumazawa, N., Three dimensional virtual crack closure-integral method (VCCM) with skewed and non-symmetric mesh arrangement at the crack front, Engineering Fracture Mechanics, Vol. 72 (2005), pp. 1717-1737.

(15) Schollmann, M., Richard, H. A., Kullmer, G., and Fulland, M., A new criterion for the prediction of crack development in multiaxially loaded structures, International Journal of Fracture, Vol. 117 (2002), pp. 129-141.

(16) Richard, H. A., Fulland, M. and Sander, M., Theoretical crack path prediction, Fatigue and Fracture of Engineering Materials and Structures, Vol. 28 (2005), pp. 3-12.

(17) Skinner Jr., J. D., Finite element predictions of plasticity-induced fatigue crack closure in three-dimensional cracked geometries, Master Thesis, Mississippi State University, 2001.

(18) Wu, Z. X., On the through-thickness crack with a curved front in center-cracked tension specimens, Engineering Fracture Mechanics, Vol. 73 (2006), pp. 1600-1613. 